\title{
Future role for adoptive T-cell therapy in checkpoint inhibitor-resistant metastatic melanoma
}

\author{
Troels Holz Borch (D) , Rikke Andersen, ${ }^{2}$ Eva Ellebaek, ${ }^{1}$ Özcan Met, ${ }^{1}$ Marco Donia, \\ Inge Marie Svane ${ }^{1}$
}

To cite: Borch TH, Andersen R, Ellebaek $\mathrm{E}$, et al. Future role for adoptive T-cell therapy in checkpoint inhibitor-resistant metastatic melanoma. Journal for ImmunoTherapy of Cancer 2020;8:e000668. doi:10.1136/ jitc-2020-000668

- Additional material is published online only. To view, please visit the journal online (http://dx.doi.org/10.1136/jitc2020-000668).

Accepted 07 April 2020

\section{ABSTRACT}

Personalized cell therapy targeting tumor antigens with expanded tumor-infiltrating lymphocytes (TILs) has shown great promise in metastatic melanoma (MM) since the 90s. However, MM was first-in line to benefit from the wave of checkpoint inhibitors (CPI), which shifted the focus of immunotherapy almost fully to immune CPI. Still, the majority of patients fail to benefit from CPI treatment, raising the intriguing question on how TIL therapy may fit into the changing landscape of melanoma treatment. We took advantage of data from a unique cohort of patients with MM treated with T-cell therapy in consecutive clinical trials at our institution across the last 10 years. Based on detailed data on patient characteristics, pre-TIL and post-TIL treatments and long-term follow-up, we were able to address the important issue of how TIL therapy can be positioned in the current CPI era. We found that previous progression on anticytotoxic T-lymphocyteassociated protein 4 do not seem to harm neither rate nor duration of response to TIL therapy. Importantly, even in the hard-to-treat population of patients who progressed on antiprogrammed cell death protein 1 (anti-PD-1), an objective response rate of $32 \%$ was achieved, including durable responses. Yet, median progression-free survival was reduced in this anti-PD-1 refractory population. Trial registration number: ClinicalTrials.gov ID: NCT00937625, NCT02379195 and NCT02354690.

\section{INTRODUCTION}

The treatment landscape of metastatic melanoma (MM) has evolved rapidly during the past decade. Multiple antibodies blocking immune regulatory receptors, so-called immune checkpoint inhibitors (CPI), and several small molecule inhibitors targeting BRAF and/or MEK (only for patients whose tumor cells harbor a BRAF mutation), have been approved by major regulatory agencies. In both clinical trials and real-life clinical data, the administration of these new treatments led to an improved survival of patients with MM, raising the bar of long-term survivors substantially. ${ }^{1-3}$ Nevertheless, most patients do not benefit or eventually progress leaving a large unmet medical need.
Experimental treatment with adoptive transfer of tumor-infiltrating lymphocytes (TIL) has demonstrated robust response rates (RR) of up to $50 \%$ with durable complete $\mathrm{RR}$ in about $15 \%$ of treated patients across multiple phase I/II trials. ${ }^{4-9}$ Most patients in published reports have received prior therapy, but only a small fraction received prior treatment with CPI. While both positive and negative effects on clinical outcome after TIL therapy has been reported for prior treatment with anticytotoxic T-lymphocyteassociated protein 4 (anti-CTLA-4), ${ }^{70}$ very few data are available for patients previously treated with antiprogrammed cell death protein 1 (anti-PD-1), antibodies and how this affects clinical response to TIL therapy is largely unknown.

To elucidate this clinically important question, we analyzed data from three different TIL trials conducted at a single institution, which included patients either not previously treated with CPI or who have progressed on or after CPI.

\section{METHODS}

\section{Patients and clinical trials}

Data from three clinical trials were pooled. All patients were treated with 7 days of lymphodepleting chemotherapy with cyclophosphamide and fludarabine, infusion of autologous TILs and interleukin-2 (IL-2). Study I was performed in two parts including the first 6 patients treated with low-dose IL-2 subcutaneously and the next 25 consecutive patients treated with an intermediate continuous intravenous IL-2 dosing regimen (decrescendo regimen) (ClinicalTrials.gov ID. In both study II and study III), 12 patients were treated with the decrescendo IL-2 regimen. Further information on clinical trial design and treatment details is available in previously published reports for study $\mathrm{I}^{611}$ and study II, ${ }^{5}$ 
and manuscript for study III is in preparation. ${ }^{12}$ Data cutoff for this analysis was 14 December 2018.

Importantly, in these trials all TILs were produced at a single institution and following similar procedures ensuring comparability of treatment products.

\section{Statistical analysis}

Survival and progression-free survival curves were computed according to the Kaplan-Meier method using GraphPad Prism V.5 software. P values of comparisons between survival curves were performed using the logrank Mantel-Cox test. RR comparisons of subgroups were tested using Fisher's exact test. A p value of $<0.05$ was considered to be statistically significant.

\section{RESULTS}

\section{Patient characteristics}

A total of 75 patients were enrolled with an intention-totreat and had a tumor lesion surgically removed between August 2009 and May 2018. For two patients (2.7\%), TIL growth was not successful. In one patient, the removed tumor was found to be a sarcoma and 17 patients $(22.7 \%)$ deteriorated clinically and were excluded before treatment with TILs. Online supplementary figure S1 shows an outline of patient enrollment and number of dropouts. Thus, a total of 55 patients were treated with TILs. For those patients, the TIL product was ready for infusion in a median of 5.5 weeks after surgery (data not shown).

At baseline, all patients had progressive MM, unresectable stage IIIB or higher with $78 \%$ being stage M1c (according to the Melanoma American Joint Commission of Cancer, AJCC staging seventh edition; table 1). All patients had received prior systemic therapy (median 2; range 1-4) for unresectable or metastatic disease, and $84 \%$ had received two or more prior lines of therapy (table 1). Most patients had widespread metastatic disease, with a median of four organ systems involved (range 1-8) including 33\% having liver metastasis and/ or a history of brain metastasis (these had to be small, with no edema and asymptomatic, or treated and stable), and $62 \%$ had elevated lactate dehydrogenase (LDH). At inclusion, all patients were in good performance status (PS 0-1), however some patients clinically deteriorated during TIL manufacturing (typically $4-6$ weeks) and at admission three patients had $\mathrm{PS} \geq 2$.

\section{Treatment characteristics and adverse events}

TIL therapy comprised preconditioning chemotherapy, infusion of autologous TILs and subsequent interleukin-2 is an intense treatment requiring admission for monitoring and managing of side effects. We observed no new safety signals, although two patients died within days after receiving TIL infusion; one patient due to an intratumoral hemorrhage in a previously irradiated brain metastasis and one patient from multiorgan failure attributed to the patient's fast progressing cancer and very large tumor burden described in more detail in Andersen et
Table 1 Baseline characteristics of treated patients

$\mathrm{n}=55 \%$

\begin{tabular}{llrl}
\hline Age (median, range) & & 53 & $(25-73)$ \\
\hline ECOG PS & 0 & 34 & 62 \\
& 1 & 18 & 33 \\
\hline Stage & IIIB & 3 & 5 \\
& M1a & 2 & 4 \\
\hline & M1b & 4 & 7 \\
\hline Elevated LDH & M1c & 43 & 11 \\
\hline BRAF mutated & & 34 & 62 \\
\hline No. of metastatic sites (median, range) & 32 & 58 \\
\hline History of brain metastasis & 4 & $(1-8)$ \\
\hline History of liver metastasis & 7 & 13 \\
\hline History of brain and liver metastasis & 7 & 13 \\
\hline Lines of prior therapy & 1 & 4 & 7 \\
\hline & 2 & 9 & 16 \\
\hline & $\geq 3$ & 28 & 51 \\
\hline Previous therapy & Interleukin-2 & 33 & 33 \\
\hline & Ipilimumab & 41 & 74 \\
\hline & Anti-PD-1 therapy & 23 & 42 \\
\hline & Temozolomide & 5 & 9 \\
\hline & BRAF inhibitor & 10 & 18 \\
\hline
\end{tabular}

ECOG, Eastern Cooperative Oncology Group; LDH, lactate dehydrogenase; PD-1, programmed cell death protein 1; PS, performance status.

$a l^{5}$ The two deaths underline that patient selection is important in the context of TIL therapy.

The median time in hospital was 19 days (online supplementary table T1). All patients experienced bone marrow suppression with the majority needing multiple red blood cell and platelet transfusions. Furthermore, most patients developed well-known side effects of IL-2 treatment (eg, electrolyte derangement, hypotension, fluid retention, fever and dyspnea). Toxicities were transient (except vitiligo observed in 3 of 55 patients) and responded to standard interventions or cessation of treatment. On discharge, all toxicities had resolved or was grade 1 or 2 .

\section{Response rate, overall survival and progression-free survival}

Of the 55 patients treated, 53 patients were evaluable according to Response Evaluation Criteria in Solid Tumors criteria. Of 53 evaluable patients, 6 patients achieved a complete response (CR) and 14 patients achieved a partial response (PR), hence the overall RR was $38 \%$ (online supplementary table T2). Two patients died within days after receiving TIL infusion and were, thus, not evaluable.

With a median follow-up time of 60 months for the entire cohort, the median overall survival (mOS) was 15.9 
A

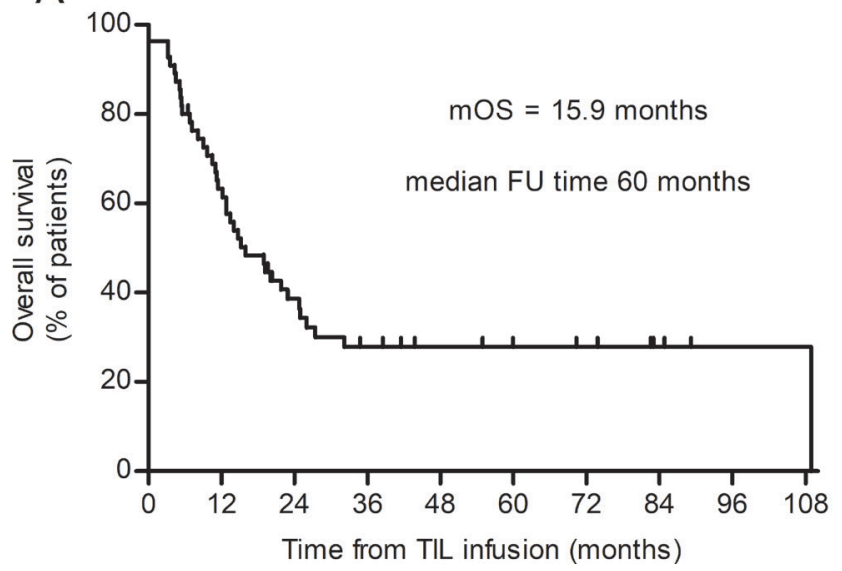

B

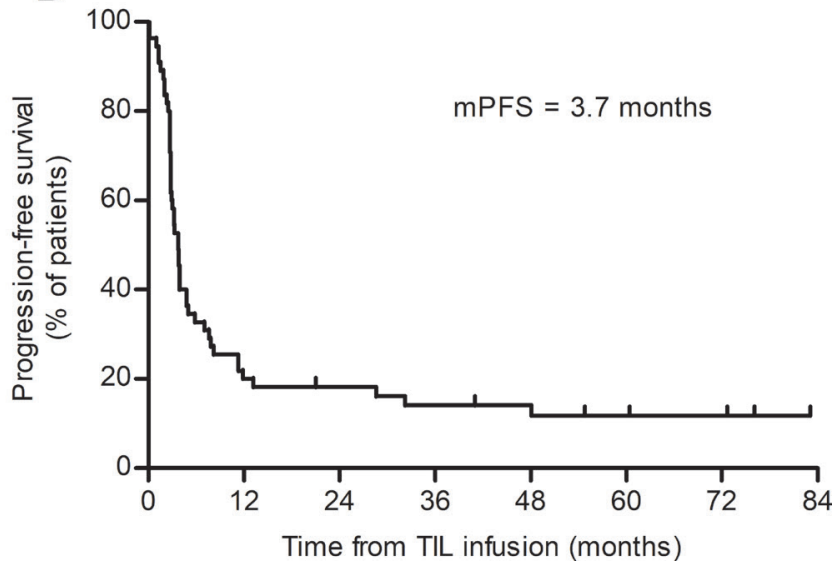

Figure 1 Median overall survival (mOS) and median progression-free survival (mPFS) after infusion of tumor-infiltrating lymphocytes (TIL). Kaplan-Meier curves showing OS (A) and PFS (B) for the entire cohort $(n=55)$.

months, with the survival curve reaching a plateau after 30 months, and a 3 -year survival rate at $29 \%$ (figure $1 \mathrm{~A}$ ).

Median progression-free survival (mPFS) was 3.7 months, nevertheless only few patients progressed later than 12 months after treatment indicating that most patients with disease control were still progression free many years later (figure 1B). Indeed, seven patients $(13 \%)$ had not progressed at data cut-off, and another patient died of an unrelated infectious disease almost 3 years after treatment.

\section{TIL therapy induces durable responses}

Of the 20 patients with a CR or PR, $13(65 \%)$ are still alive. Hence, mOS was not reached for this subgroup and mPFS was 19.1 months (see figure $2 \mathrm{~A}$ and $\mathrm{B}$ ).

Of the six complete responders, two patients progressed after 13.2 and 48.1 months, but had solitary relapses removed and one is still without evidence of disease (NED) 76+ months after surgery. The 3-year survival rate for complete responders was $100 \%$ (data not shown). Of interest, the two patients progressing after CR both received low-dose IL-2 in the first part of study I, whereas the four remaining complete responders (ongoing

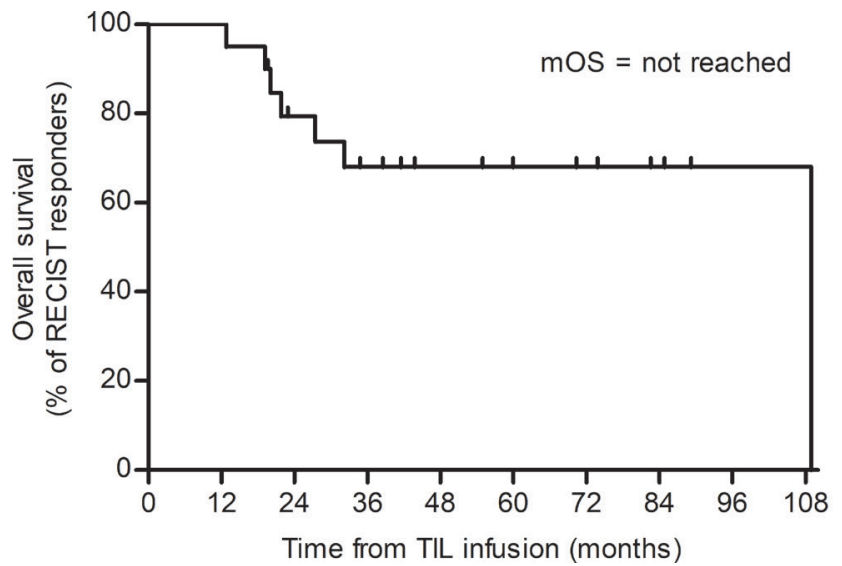

between $41+$ and $83+$ months) received an intermediate IL-2 dosing regimen.

Three patients with PR have not progressed. Two of these had residual disease removed surgically and have NED as previously described, ${ }^{11}$ now $73+$ and $76+$ months after treatment.

\section{Influence of prior checkpoint inhibition on TIL therapy outcome}

Patients with tumors harboring certain immunohistopathological features (ie, dense immune cell infiltration, high mutational burden and interferon (IFN)- $\gamma$ signature) have a higher chance of responding to CPI. ${ }^{13}$ This implies a selection towards enrichment of less favorable characteristics in patients progressing on or after treatment with CPI. In the present cohort, most patients had received and progressed on prior treatment with CPI, and to elucidate whether resistance to checkpoint inhibition affects clinical outcome after TIL therapy, we performed subgroup analyzes stratifying for prior treatment with either anti-CTLA-4 only, anti-PD-1 only, sequential antiCTLA-4 and anti-PD-1, or no prior CPI. Patients were only stratified for prior CPIs, but the majority had received

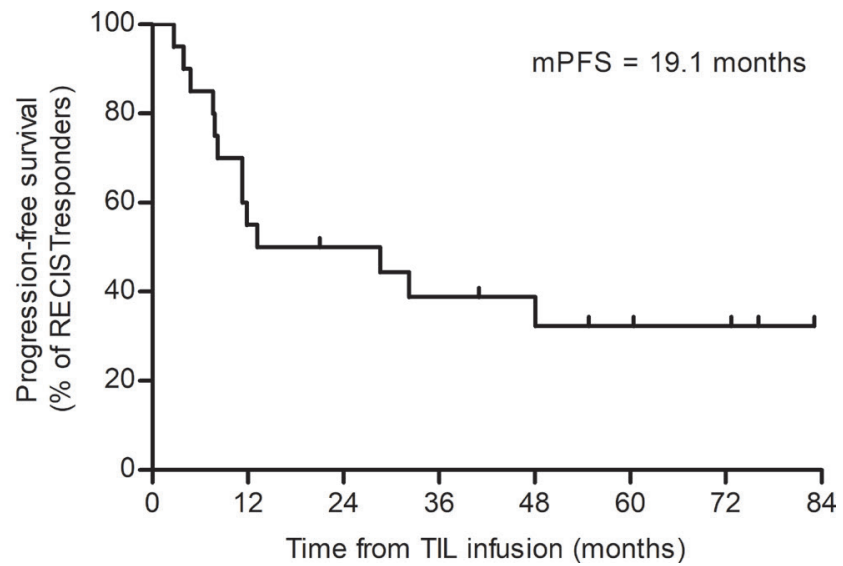

Figure 2 Median overall survival (mOS) and median progression-free survival (mPFS) for Response Evaluation Criteria in Solid Tumors (RECIST) responders. Kaplan-Meier curves showing OS (A) and PFS (B) for RECIST responders ( $n=20)$. TIL, tumorinfiltrating lymphocytes. 
A

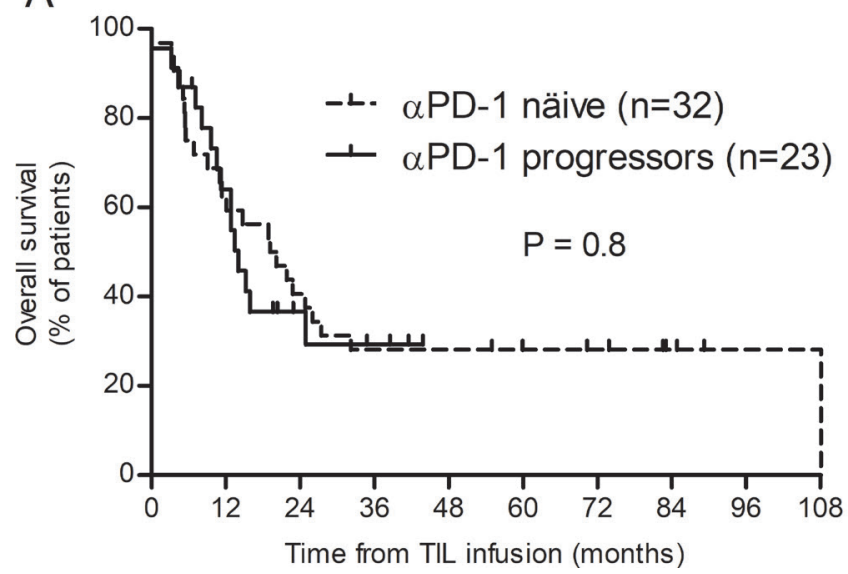

B

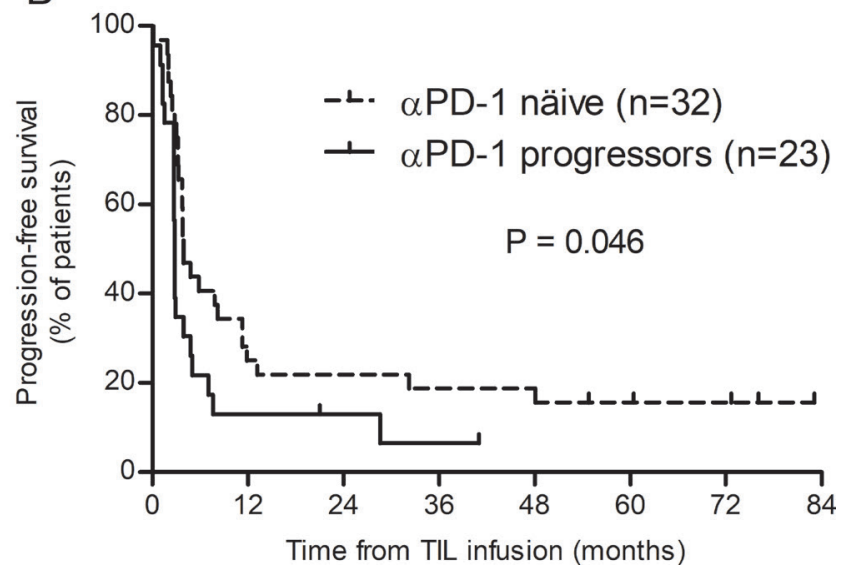

Figure 3 Overall survival (OS) and progression-free survival (PFS) according to antiprogrammed cell death protein 1 (antiPD-1) status prior to tumor-infiltrating lymphocytes (TIL) therapy. Kaplan-Meier curves showing OS (A) and PFS (B) in anti-PD-1näive patients $(n=32)$ or patients previously progressed on anti-PD-1 blockade $(n=23)$. Dotted lines represent SE.

other systemic lines of treatment in addition. The number of prior lines of therapy were well balanced except for the group of patients treated with anti-PD-1 only $(n=4)$, as they all received TIL therapy as a second-line therapy (data not shown).

In our cohort, a total of 41 patients had received prior treatment with anti-CTLA-4. Of those, 22 patients had received no other prior CPI and this subgroup appeared to have a longer mOS compared with patients who were not previously treated with CPI (21.8 vs 11.2 months), but survival curves were not significantly different $(p=0.5)$ and plateau was reached at the same time and level (online supplementary figure S2A). The same pattern was observed for PFS (online supplementary figure S2B). RR was numerically higher but not statistically significant in the prior anti-CTLA-4-treated group ( $45.5 \%$ vs $33.3 \%$; $\mathrm{p}=0.48$ ).

Results from published studies indicated that response to TIL therapy after progression on anti-PD-1 is possible, although RR appeared low. ${ }^{10}{ }^{15}$ In our cohort, a total of 23 patients had received prior treatment with anti-PD-1 either as the only CPI $(n=4)$ or sequentially to anti-CTLA-4 $(n=19)$. To evaluate specifically the effect of anti-PD-1, the group of patients previously treated with anti-CTLA-4 alone was compared with patients treated with anti-PD-1 and anti-CTLA-4 sequentially. Clinical features and prognostic variables (PS, stage, LDH, metastatic sites, prior lines of therapy) were similar (data not shown). PFS curves for the two groups were significantly different favoring the patients previously treated with anti-CTLA-4 alone ( $\mathrm{p}=0.03$; mPFS 4.9 vs 2.8 months), but no significant difference between the groups was observed for OS $(\mathrm{p}=0.56)$ and $\mathrm{RR}(\mathrm{p}=0.52)$ (online supplementary figure S3 and table T2).

As prior treatment with anti-CTLA-4 did not significantly affect clinical response after TIL therapy, we divided our data set in patients previously treated with anti-PD-1 regardless of anti-CTLA-4 treatment (PD-1 progressors; $n=23$ ), or not (PD-1 naïve; $n=32$ ). Baseline clinical characteristics were similar between the two groups (online supplementary table T3). No statistical difference was observed in OS and RR (figure 3A and table 2), but PFS curves started to separate at around 4 months and are significantly different favoring the antiPD-1-naïve group ( $p=0.046$, figure $3 \mathrm{~B}$ ). The difference appeared to be driven by a longer median duration of response (DOR) in the anti-PD-1-naïve group as a whole (32.2 vs 7.6 months) and especially for partial responders (table 2).

Although not systematically assessed, it is noteworthy that durable responses to anti-PD-1 were observed in two anti-PD-1-naïve patients who progressed after TIL therapy (one patient had PR and one had PD on TIL).

\section{Prior treatments influence the duration of response}

Experimental treatments like TIL therapy are often employed only after progression on approved therapies. Since multiple therapies have been approved for MM during the last decade, TIL therapy has been moved to third or fourth line of treatment depending on BRAF mutational status. To assess the impact of moving TIL therapy to later lines, we analyzed treatment responses according to line of treatment. In our cohort, all patients were treated in second line or later. We found that patients treated in second line responded favorably with a RR of $62.5 \%$ compared with $33.3 \%$ for later lines and had numerically higher median OS and PFS, although not statistically significant (online supplementary table $\mathrm{T} 4$ and figure $\mathrm{S} 4$ ). CR rates were significantly impacted by the line of treatment as $33.3 \%$ (3/9) achieved CR if treated in second line compared with $6.5 \%(3 / 46)$ treated in third or later line $(\mathrm{p}=0.049)$.

Median DOR decreased incrementally with increasing number of prior lines of therapy and, notably, only one of 18 patients treated in fourth line or later have an ongoing response (PR (NED); (online supplementary table T4). 
Table 2 Clinical response to TIL therapy in PD-1-naïve patients vs PD- 1 progressors

\begin{tabular}{|c|c|c|}
\hline & No prior $\alpha$ PD-1 & $\begin{array}{l}\text { Prior } \alpha \text { PD- } \\
1 \pm \alpha C T L A-4\end{array}$ \\
\hline & $n=32$ & $n=23$ \\
\hline Response rate $^{\star}$ & $42 \%$ & $32 \%$ \\
\hline Median OS (months) & 19.6 & 14 \\
\hline Median PFS (months) & 3.9 & 2.8 \\
\hline $\begin{array}{l}\text { DOR both CR and PR, } \\
\text { median (months) }\end{array}$ & 32.2 & 7.6 \\
\hline \# CR & 5 & 1 \\
\hline DOR median (months) & 54.8 & $41 \ddagger$ \\
\hline DOR min (months) & 13.2 & $41 \ddagger$ \\
\hline DOR max (months) & 83.1 & $41 \ddagger$ \\
\hline DOR range (months) & (13.2-83.1‡) & $(41 \ddagger)$ \\
\hline \# PR & 8 & 6 \\
\hline DOR median (months) & 11.3 & 6.2 \\
\hline DOR min (months) & 7.8 & 2.7 \\
\hline DOR max (months) & $76.1 \ddagger$ & 28.6 \\
\hline DOR range (months) & $(7.8-76.1 \ddagger)$ & $(2.7-28.6)$ \\
\hline $\begin{array}{l}\text { \# Ongoing responses } \\
\text { (CR/PR) }\end{array}$ & $5(3 / 2)$ & $2(1 / 1)$ \\
\hline
\end{tabular}

*One patient in each group was not evaluable for response. †Duration of response. fOngoing response.

$\mathrm{CR}$, complete response; DOR, duration of response; OS, overall survival; PD-1, programmed cell death protein 1; PFS, progressionfree survival; PR, partial response; TIL, tumor-infiltrating lymphocytes.

\section{DISCUSSION}

In the present study, we pooled data from three clinical TIL therapy trials including patients not previously treated with CPI or who had progressed on one or two lines of CPI. In this heavily pretreated cohort, we found a RR of $38 \%$ which is in line with previous reports. ${ }^{47810}$

In particular, CR after TIL therapy has been reported to be durable, ${ }^{410}$ which is corroborated by our data $(4 / 6$ CRs ongoing). Of interest, the two patients achieving CR with later recurrence both received low-dose subcutaneous IL-2. Whether there is a biological effect behind this observation, cannot be discerned from these data. The question is unlikely to be answered in the near future as attempts to perform randomized testing have either failed to enroll patients (NCT01995344) or was terminated early due to meeting prespecified futility boundaries as reported by Amaria et al at ASCO 2019. ${ }^{16}$ However, the only patient with ongoing PR in that trial received low-dose IL-2.

Despite the recurrences, both patients with CR after low-dose IL-2 did not require another systemic treatment for more than $>4$ years and not to date, respectively. Lowering the dose of IL-2 is intriguing as side effects are dose-dependent, ${ }^{17}$ and ability to tolerate IL-2-related toxicity is one of the factors demanding proper patient selection for TIL therapy.

Clinical response to TIL therapy has been reported to be both negatively and positively impacted by prior treatment with anti-CTLA-4. ${ }^{70}$ While our data suggest a numerically slightly higher RR and longer mOS in patients previously treated with anti-CTLA- 4 and not antiPD-1, we did not find any clinical meaningful advantage, but at least no detrimental effects were observed.

It has been shown that patients with tumors harboring favorable histopathological characteristics such as dense immune cell infiltration, high mutational burden and IFN- $\gamma$ signature are more likely to respond to anti-PD-1. ${ }^{1314}$ The implication being that tumors progressing on or after anti-PD-1 are enriched with less favorable histopathological characteristics. We found the RR to be unaffected by previous anti-PD-1. However, DOR was lower in anti-PD-1 progressors, at least for partial responders, which corresponds well with previously published results. ${ }^{10}{ }^{15}$ Similar results were presented at ASCO 2019 by Sarnaik et al, ${ }^{18}$ with a RR of $38 \%$ in 55 patients progressing after antiPD-1 (most had also received anti-CTLA-4 and BRAF/ MEK inhibitors if BRAF mutated). Follow-up in that study is still short and median DOR has not been reached.

Our observation that TIL therapy can induce clinical responses in an anti-PD-1 refractory patient cohort suggests that resistance to the individual therapies is at least partially mediated by independent mechanisms. This is further corroborated by two anti-PD-1-naïve patients progressing after TIL in this dataset, later achieving clinical response on anti-PD-1-as also observed by others. ${ }^{10} 15$ We have previously shown that T-cell infiltration in antiPD-1-resistant tumors was diverse ranging from almost none to dense, but tumor-reactive $\mathrm{T}$ cells could be grown from most of these tumors. ${ }^{5}$ Furthermore, we have shown TIL resistance can be mediated by defects in the antigen presentation machinery, ${ }^{19}$ which would likely also confer resistance to CPI. Resistance mechanisms to TIL therapy is an area that needs further investigation in order to rationally design new trials and lift the 'tail' of the survival curve.

Not surprisingly, our data show a favorable RR for patients treated in second line compared with patients treated in third or later line and median DOR in later lines is decreasing incrementally. As there are now multiple lines of approved therapies for MM, TIL therapy is often moved to later lines. Considering the high response rate and curative potential, perhaps this strategy should be challenged. Patients for TIL therapy undergo considerable selection as they need to have a surgically resectable tumor, TILs must be capable of growth, the patient should be able to wait $\sim 6$ weeks for treatment and must be able to tolerate IL-2. In the present study, there was an attrition rate of 27\% (20 of 75), which is comparable to an intention-to-treat report from Besser $e t a l^{8}$ Even with this selection in mind and despite the obvious limitation in comparison between studies, TIL therapy after progression on anti-PD-1 seems to be at least non-inferior to either treatment with ipilimumab or 
ipilimumab and nivolumab in combination with reported RRs of $4 \%-16 \%$ and $20 \%-21 \%$, respectively. ${ }^{20-22}$ Comparison of ipilimumab and TIL therapy in a first-line or secondline setting is formally tested in an ongoing randomized phase III trial (NCT02278887). To our knowledge, response to TIL therapy after combined anti-CTLA- 4 and anti-PD-1 has not been reported in sufficient numbers to draw any conclusions.

It is also important to compare the treatment characteristics and toxicity profile of TIL therapy with other available therapies relevant in second line. All patients developed transient grade 3-4 adverse events during the 3 weeks in hospital due to high-dose chemotherapy and IL-2. These events were expected and managed by established treatment algorithms and on discharge all toxicities had resolved or were grade 1-2. Only vitiligo in 5\% of patients was observed as a permanent toxicity. This is contrast to treatment with CPI, which is administered on an outpatient basis potentially for up to 2 years and a comparably smaller, yet significant, proportion of patients experiencing grade 3-4 adverse events (28\% for ipilimumab, $59 \%$ for the combination of nivolumab and ipilimumab). ${ }^{1}$ Also significant is the risk of long-term toxicity, primarily endocrine events, requiring life-long hormonal replacement therapy. Thus, both treatment characteristics and toxicity profiles differ and could be a selection parameter for patients themselves and for treating physicians.

A challenge in TIL therapy is attrition due to TIL production time. This has been addressed by multiple centers by 'bridging' with either BRAF inhibitor treatment $^{23}{ }^{24}$ or ipilimumab ${ }^{25}$ with both strategies being feasible in not adding toxicity and keeping attrition to a minimum while maintaining a high response rate. Another approach is combining TIL therapy with anti-PD-1 either in PD-1 progressors (multiple academic centers) or in PD-1-naïve patients as pursued in a commercial setting (NCT03645928).

\section{CONCLUSION}

TIL therapy requires selection of patients for treatment, but even in this hard-to-treat population of patients with MM who progressed on anti-PD-1, with or without antiCTLA sequentially, an objective response rate of $32 \%$ was achieved and included durable responses.

These observations warrant further use of and research in TIL therapy.

Contributors THB and IMS designed the study, interpreted the data, analyzed the results and wrote the manuscript. RA, EE, ÖM and MD assisted with manuscript review and revision. All authors gave their final approval of the manuscript submitted.

Funding The clinical trials were funded through grants from The Danish Cancer Society, Aase and Einar Danielsen Foundation, The Beckett Foundation, Herlev and Gentofte Hospital Research Council and The Danish Council for Independent Research.

Competing interests THB has received travel support from Roche and MSD, and travel support and speaker's fee from Bristol-Myers Squibb (BMS). EE has received honorarium from Roche and BMS, and travel support from MSD and BMS. MD has received honorarium from Genzyme, Merck Sharp \& Dohme (MSD) and BMS, and travel support from Novartis, MSD, BMS, Roche and Pfizer. IMS has an advisory board relationship with or lectured for Roche, Novartis, MSD, Celgene, Incyte, TILT bio, Pfizer, BMS and AstraZeneca, and has received limited grants for translational research from BMS, Roche and Novartis. RA and ÖM report no conflicts of interest.

\section{Patient consent for publication Not required.}

Ethics approval Ethics approval was granted by the National Committee on Health Research Ethics for each of the clinical trials, and informed consent to participate was obtained from all subjects.

Provenance and peer review Not commissioned; externally peer reviewed.

Data availability statement Data are available on reasonable request from the corresponding author.

Open access This is an open access article distributed in accordance with the Creative Commons Attribution Non Commercial (CC BY-NC 4.0) license, which permits others to distribute, remix, adapt, build upon this work non-commercially, and license their derivative works on different terms, provided the original work is properly cited, appropriate credit is given, any changes made indicated, and the use is non-commercial. See http://creativecommons.org/licenses/by-nc/4.0/.

\section{ORCID iD}

Troels Holz Borch http://orcid.org/0000-0002-4402-9281

\section{REFERENCES}

1 Larkin J, Chiarion-Sileni V, Gonzalez R, et al. Five-Year survival with combined nivolumab and ipilimumab in advanced melanoma. $N$ Engl J Med 2019;381:1535-46.

2 Ugurel S, Röhmel J, Ascierto PA, et al. Survival of patients with advanced metastatic melanoma: the impact of novel therapiesupdate 2017. Eur J Cancer 2017;83:247-57.

3 Donia M, Ellebaek E, Øllegaard TH, et al. The real-world impact of modern treatments on the survival of patients with metastatic melanoma. Eur J Cancer 2019;108:25-32.

4 Rosenberg SA, Yang JC, Sherry RM, et al. Durable complete responses in heavily pretreated patients with metastatic melanoma using T-cell transfer immunotherapy. Clin Cancer Res 2011;17:4550-7.

5 Andersen R, Borch TH, Draghi A, et al. T cells isolated from patients with checkpoint inhibitor-resistant melanoma are functional and can mediate tumor regression. Ann Oncol 2018;29:1575-81.

6 Ellebaek E, Iversen TZ, Junker N, et al. Adoptive cell therapy with autologous tumor infiltrating lymphocytes and low-dose interleukin-2 in metastatic melanoma patients. J Trans/ Med 2012;10:169.

7 Forget M-A, Haymaker C, Hess KR, et al. Prospective analysis of adoptive TIL therapy in patients with metastatic melanoma: response, impact of anti-CTLA4, and biomarkers to predict clinical outcome. Clin Cancer Res 2018;24:4416-28.

8 Besser MJ, Shapira-Frommer R, Itzhaki O, et al. Adoptive transfer of tumor-infiltrating lymphocytes in patients with metastatic melanoma: intent-to-treat analysis and efficacy after failure to prior immunotherapies. Clin Cancer Res 2013;19:4792-800.

9 Dafni U, Michielin O, Lluesma SM, et al. Efficacy of adoptive therapy with tumor-infiltrating lymphocytes and recombinant interleukin-2 in advanced cutaneous melanoma: a systematic review and metaanalysis. Ann Oncol 2019;30:1902-13.

10 Goff SL, Dudley ME, Citrin DE, et al. Randomized, prospective evaluation comparing intensity of lymphodepletion before adoptive transfer of tumor-infiltrating lymphocytes for patients with metastatic melanoma. J Clin Oncol 2016;34:2389-97.

11 Andersen R, Donia M, Ellebaek E, et al. Long-Lasting complete responses in patients with metastatic melanoma after adoptive cell therapy with tumor-infiltrating lymphocytes and an attenuated IL2 regimen. Clin Cancer Res 2016;22:3734-45.

12 Borch TH, Andersen R, Rana MAH, et al. Clinical efficacy of T-cell therapy after short-term BRAF-inhibitor induction in checkpoint inhibitor resistant metastatic melanoma patients. Annals of Oncology 2018;29:viii402.

13 Cristescu R, Mogg R, Ayers M, et al. Pan-tumor genomic biomarkers for PD-1 checkpoint blockade-based immunotherapy. Science 2018;362. doi:10.1126/science.aar3593. [Epub ahead of print: 12 Oct 2018].

14 Tumeh PC, Harview CL, Yearley JH, et al. Pd-1 blockade induces responses by inhibiting adaptive immune resistance. Nature 2014;515:568-71.

15 Nguyen LT, Saibil SD, Sotov V, et al. Phase II clinical trial of adoptive cell therapy for patients with metastatic melanoma with autologous 
tumor-infiltrating lymphocytes and low-dose interleukin-2. Cancer Immunol Immunother 2019;68:773-85.

16 Amaria RN, Haymaker CL, Forget M-A, et al. Lymphodepletion (LD), tumor-infiltrating lymphocytes (TIL) and high (HD-IL2) versus lowdose (LD-IL2) IL-2 followed by pembrolizumab (pembro) in patients (PTS) with metastatic melanoma (Mm). JCO 2019;37:9543-5.

17 Schwartzentruber DJ. Guidelines for the safe administration of highdose interleukin-2. J Immunother 2001;24:287-93.

18 Sarnaik A, Khushalani NI, Chesney JA, et al. Safety and efficacy of cryopreserved autologous tumor infiltrating lymphocyte therapy ( LN-144, lifileucel) in advanced metastatic melanoma patients who progressed on multiple prior therapies including anti-PD-1. JCO 2019;37:2518-9.

19 Donia M, Harbst K, van Buuren M, et al. Acquired immune resistance follows complete tumor regression without loss of target antigens or IFN $\gamma$ signaling. Cancer Res 2017;77:4562-6.
20 Zimmer L, Apuri S, Eroglu Z, et al. Ipilimumab alone or in combination with nivolumab after progression on anti-PD-1 therapy in advanced melanoma. Eur J Cancer 2017;75:47-55.

21 Weichenthal M, Ugurel S, Leiter UM, et al. Salvage therapy after failure from anti-PD-1 single agent treatment: a study by the German ADOReg melanoma registry. JCO 2019;37:9505-6.

22 Long G, Robert C, Blank C, et al. Outcomes in patients (PTS) treated with ipilimumab (IPI) after pembrolizumab (pembro) in KEYNOTE-006. Pigment Cell Melanoma Res 2017;30:76-156.

23 Atay C, Kwak T, Lavilla-Alonso S, et al. Braf targeting sensitizes resistant melanoma to cytotoxic T cells. Clin Cancer Res 2019;25:2783-94.

24 Deniger DC, Kwong MLM, Pasetto A, et al. A pilot trial of the combination of vemurafenib with adoptive cell therapy in patients with metastatic melanoma. Clin Cancer Res 2017;23:351-62.

25 Mullinax JE, Hall M, Prabhakaran S, et al. Combination of ipilimumab and adoptive cell therapy with tumor-infiltrating lymphocytes for patients with metastatic melanoma. Front Oncol 2018;8:44. 